\title{
NOTE ON A PAPER OF DIEUDONNE
}

\section{CARLITZ}

In a recent paper [1], Dieudonné has proved the following theorem.

Let $a_{0}, a_{1}, a_{2}, \cdots$ be an infinite sequence of rational numbers and consider the (formal) power series

$$
\exp \left(\sum_{m=0}^{\infty} a_{m} x^{p^{m}}\right)=\sum_{n=0}^{\infty} c_{n} x^{n}
$$

where $p$ is a fixed prime. Then a necessary and sufficient condition that all the coefficients $c_{n}$ be p-adic integers is that for each $i \geqq 0$ we have

$$
a_{i}=\frac{a_{i-1}}{p}+b_{i} \quad\left(a_{-1}=0\right),
$$

where each $b_{i}$ is a p-adic integer.

In this note we consider the following problem. Let $a_{1}, a_{2}, a_{3}, \cdots$ be a sequence of rational numbers and define the (formal) power series

$$
\exp \left(\sum_{m=1}^{\infty} a_{m} x^{m}\right)=\sum_{n=0}^{\infty} c_{n} x^{n}
$$

Then we prove the following

THEOREM 1. A necessary and sufficient condition that all the coeffcients $c_{n}$ be rational integers is that for all $k \geqq 1$ we have

$$
\sum_{r s=k} r a_{r} \mu(s) \equiv 0(\bmod k),
$$

where $\mu(s)$ is the Möbius function.

To prove the theorem we employ a device used by Schur [2] and credited to Jänichen. Let

$$
g(x)=\sum_{n=0}^{\infty} c_{n} x^{n}
$$

Then we can recursively determine $b_{1}, b_{2}, \cdots$ so that

$$
g(x)=\prod_{m=1}^{\infty}\left(1-x^{m}\right)^{b_{m}} .
$$

Received by the editors June 3, 1957. 
Moreover the $b_{m}$ are all integral if and only if the $c_{n}$ are all integral. Now from (1) and (3) it follows that

$$
\begin{aligned}
\sum_{k=1}^{\infty} a_{k} x^{k} & =\log g(x)=-\sum_{m=1}^{\infty} b_{m} \sum_{r=1}^{\infty} \frac{x^{m r}}{r} \\
& =-\sum_{k=1}^{\infty} \frac{x^{k}}{k} \sum_{m r=k} m b_{m} ;
\end{aligned}
$$

consequently

$$
k a_{k}=-\sum_{m \mid k} m b_{m}
$$

But (4) is equivalent to

$$
-b_{k}=\frac{1}{k} \sum_{r s=k} r a_{r} \mu(s) .
$$

As already noted, the $c_{n}$ are all integral if and only if the $b_{k}$ are all integral, that is, if and only if the right member of (5) is integral for all $k$. But this is the same as the condition (2). This evidently proves the theorem.

The theorem can be stated in a slightly more general way. Let $P$ denote an arbitrary set of rational primes and let $D$ denote the set of rational numbers whose denominators contain only the primes of $P$. Then we may state

THEOREM 2. A necessary and sufficient condition that all the coeffcients $c_{n} \in D$ is that for all $k \geqq 1$

$$
\left\{\frac{1}{k} \sum_{r s=k} r a_{r} \mu(s)\right\} \in D .
$$

Dieudonné's theorem is obtained when $P$ consists of all the primes except $p$ and $a_{m}=0$ except for $m=p^{r}$.

\section{REFERENCES}

1. J. Dieudonné, On the Artin-Hasse exponential series, Proc. Amer. Math. Soc. vol. 8 (1957) pp. 210-214.

2. I. Schur, Arithmetische Eigenschaften der Potenzsummen einer algebraischen Gleichung, Compositio Math. vol. 4 (1937) pp. 432-444.

DUke University 